\title{
Nonsurgical treatment of infratentorial subdural empyema: A case report
}

\author{
Y. Sogoba ${ }^{1 *}$, D. Kanikomo ${ }^{1}$, O. Coulibaly ${ }^{1}$, K. Singaré ${ }^{2}$, Y. Maiga $^{3}$, D. Samaké ${ }^{2}$ S. K. Timbo ${ }^{2}$ \\ ${ }^{1}$ Department of Neurosurgery, Gabriel TOURE Hospital, Bamako, Mali; *Corresponding Author: sogobayoussouf@yahoo.fr \\ ${ }^{2}$ Department of Ear, Nose and Throat, Gabriel TOURE Hospital, Bamako, Mali \\ ${ }^{3}$ Department of Neurology, Gabriel TOURE Hospital, Bamako, Mali
}

Received 26 June 2013; revised 10 July 2013; accepted 19 July 2013

Copyright (C) 2013 Y. Sogoba et al. This is an open access article distributed under the Creative Commons Attribution License, which permits unrestricted use, distribution, and reproduction in any medium, provided the original work is properly cited.

\begin{abstract}
Infratentorial subdural empyemas are rare. It is an important neurological infection requiring immediate neurosurgical treatment. The nonsurgical treatment of subdural empyema has been reported sporadically. In this paper the authors report the nonsurgical treatment of a case of infratentorial subdural empyema. The patient with left recurrent otitis was hospitalized with symptoms of headache and fever of 3 weeks duration. Examination revealed that the patient had Glasgow Coma Scale score of 15, fever, mild cerebellar signs, no focal deficit, and abundant suppuration from the left ear. A contrast-enhanced CT scan showed an infratentorial supracerebellar hypodense fluid collection with the peripheral rim enhancement to the left of the midline that mimicked a subdural empyema. Routine hematological investigation revealed polymorphic leukocytosis and elevated erythrocyte sedimentation rate. After the left mastoidectomy and antibiotic treatment, the patient recovered with complete resolution of the subdural empyema on CT scan.
\end{abstract}

Keywords: Subdural Empyema; Otitis; Infratentorial Lesion; Antibiotic Therapy

\section{INTRODUCTION}

Subdural empyema is defined as a collection of pus in the preformed space between the cranial dura mater and arachnoid mater [1]. It is a serious intracranial infection, and in most cases prompt evacuation of the pus collection is required. Infratentorial subdural empyemas (ISDEs) are rare, constituting only $0.6 \%$ of all cases of in- tracranial suppurative disorders [2]. In this paper, the authors report a case of ISDE successfully treated with antibiotic therapy only after the left mastoidectomy had been performed.

\section{CASE REPORT}

This 24-year-old woman had been treated for left recurrent chronic otitis for years. She was hospitalized in the Ear, Nose, and Throat Department (ENT) of Gabriel TOURE Hospital with symptoms of headache and fever of 3 weeks duration. Examination revealed that the patient had a Glasgow Coma Scale (GCS) score of 15, fever, mild cerebellar signs, no focal deficit, and abundant suppuration from the left ear. A contrast-enhanced CT scan (Figure 1) showed an infratentorial supracerebellar hypodense fluid collection with peripheral rim enhancement to the left of the midline that mimicked a subdural empyema. Routine hematological investigation revealed polymorphic leukocytosis and elevated erythrocyte sedimentation rate. A left mastoidectomy was performed and culture of pus was sterile and the patient was then transferred to the department of Neurosurgery. As the lesion was thought small enough to be amenable to medical

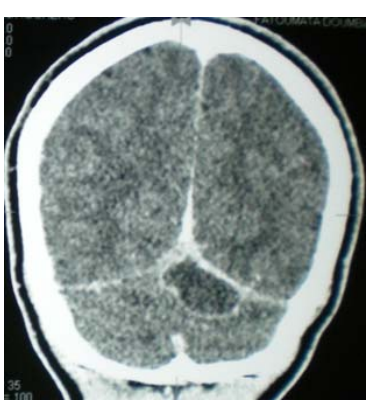

(a)

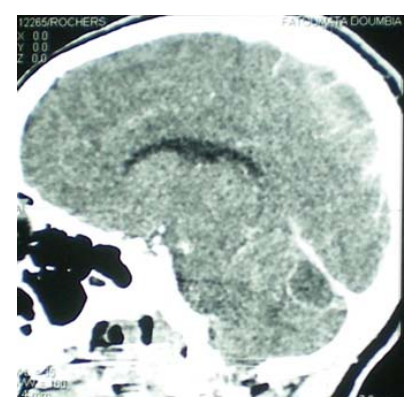

(b)
Figure 1. Coronal (a) and sagittal (b) contrast-enhanced CT scans showing the infratentorial subdural empyema. 
therapy, the patient was started on a 4-week course of empirical intravenous antibiotics including third generation of cephalosporin, metronidazole and ciprofloxacin. By the end of this course her neurological symptoms had recurred. The second CT scan (Figure 2) showed a partial resolution of the empyema and the hematological investigation became normal. The patient was then continued on a 6-week course of oral antibiotics followed by an uneventful recovery and the third CT scan (Figure 3) showed a complete resolution of the empyema. The patient was discharged home asymptomatic.

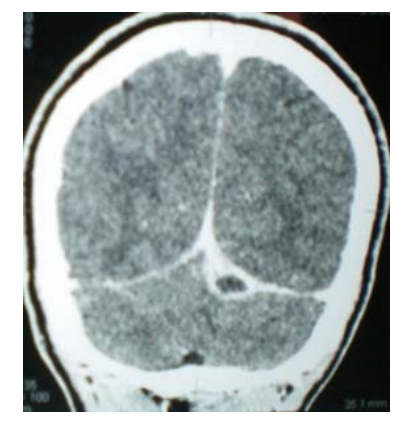

(a)

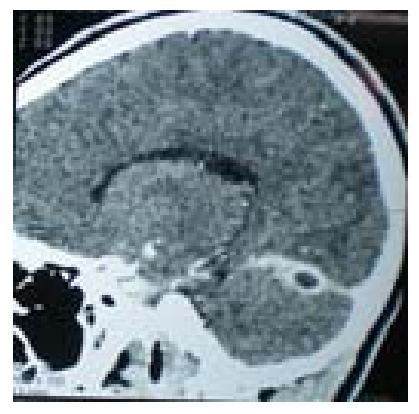

(b)

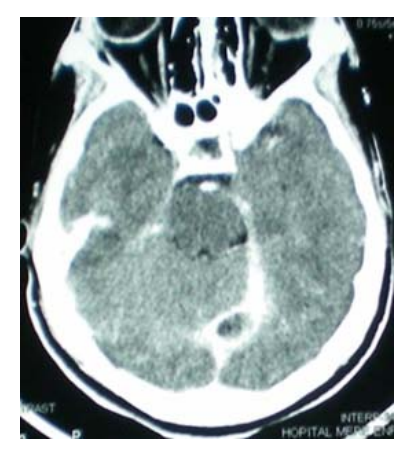

(c)

Figure 2. Coronal (a), sagittal (b) and axial (c) contrast-enhanced CT scans showing the partial resolution of the infratentorial subdural empyema after 4-week course of intravenous antibiotics treatment.

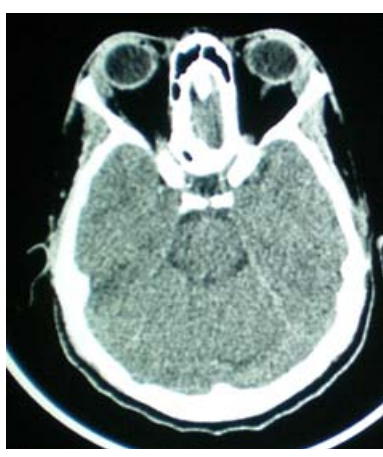

(a)

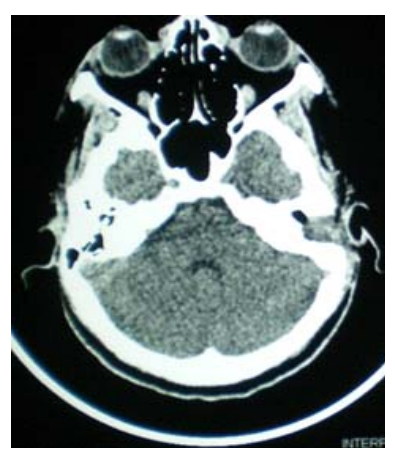

(b)
Figure 3. Axial CT scans (a) and (b) showing the complete resolution of infratentorial subdural empyema after 10 weeks of antibiotics treatment.

\section{DISCUSSION}

Subdural empyema is defined as a collection of pus in the preformed space between the cranial dura mater and arachnoid mater [1]. It is rare in the developed world due to early and judicious use of antibiotics. It remains; however, a relatively common disease entity in developing countries [3-6]. Subdural empyema represents approximately $20 \%$ of all intracranial suppurations [7]. Although pus may localize anywhere in the subdural space following ear infection or paranasal sinusitis, there is a paucity of literature regarding the infratentorial localization of pus. Morgan and Williams [8] reported a series of seven cases of posterior fossa subdural empyema that occurred during a 30-year period, and Borovich and associates [9] identified three cases of infratentorial subdural empyema over 10 years. The tendency for a greater incidence of infratentorial empyema among male patients was observed by several authors $[2,6,8,9]$. The reason for this preponderance among male patients is not known. That tendency was not noted in our case. Clinical manifestations are due to increases in intracranial pressure, focal disturbances of brain function, and constitutional symptoms due to infection $[1,6]$. The illness is usually characterized by fever, headache, vomiting, and meningism [2,8,9]. As noted by Borovich and associates [9], in cases of ISDE, the patient's clinical condition may deteriorate rapidly, and the duration of symptoms is usually shorter than in cases of supratentorial empyema. Our patient was in good neurological condition with GCS score of 15 . In the vast majority of patients with ISDE, the lesion develops as a result of chronic suppurative otitis media. Therefore the history of otorrhea should prompt further investigation even when the patient presents with nonspecific symptoms. All three patients in the series reported by Borovich and associates [9] and 71.4\% in the series reported by Morgan and Williams [8] had ISDE secondary to chronic otogenic sepsis. Our patient had left recurrent chronic otitis for years leading to ISDE. Extension of infection from the ear into the infratentorial compartment may be direct or indirect. Direct extension of infection by erosion of the bone typically causes epidural abscesses and bone infections, whereas indirect extension via progressive thrombophlebitis of the perforating blood vessels from the middle ear mucosa would typically lead to subdural empyemas and cerebellar abscesses. Apart from the otic source, other sources are well described in the literature [7,10-13], these include trauma and paranasal sinusitis. CT scan may be the most cost-effective imaging modality in subdural empyema because of its accessibility and sensitivity [3,14]. Magnetic resonance imaging, if available in the acute setting, may be the imaging modality of choice as it provides a better anatomical delineation of any collections present than does CT 
scanning, and it can adequately display areas of localized meningeal infection [14,15].

Historically, the most significant determinants of outcome in patients with subdural empyema have been aggressive early removal of the source of infection, drainage of the pus and treatment of the infection with the appropriate antibiotic medications [7,16-19].

The purpose of neurosurgical treatment is to decrease the toxic and inflammatory influences on the brain and its blood supply and to diminish the mass effect of the subdural pus and obtain pus for isolation of the causative organism and identification of its antibiotic sensitivity. Surgical treatment can involve drainage via either burr holes or craniotomy [20]. The choice of procedure has been the subject of much debate. The advantages of aspiration via burr holes are that it is simple, and it has less potential morbidity than surgical trauma. On the other hand, several reports have advocated craniotomy as the procedure of choice because it is often followed by a lower incidence of recurrence and shorter hospitalization $[16,19]$. Nonsurgical treatment is contrary to the accepted rule that a subdural empyema should be operated as soon as the diagnosis is made. Two main factors prompted us to withhold neurosurgical treatment from this patient. First, he was in good neurosurgical condition with a GCS score of 15 and limited collection of pus on CT scans. Second, our patient's rapid clinical and radiological improvement provided a strong argument for continuing medical treatment. The patient must be followed up closely clinically and radiologically when a subdural empyema is managed medically. The nonsurgical treatment of subdural empyema has been reported sporadically [21,22]. Early mastoidectomy will prevent recurrence of the empyema and development of other empyemas [13]. Therefore consultation with otorhinolaryngological colleagues is recommended as soon as possible in the course of the disease.

\section{CONCLUSION}

Although surgery with antibiotic therapy constitutes the mainstay of treatment of infratentorial subdural empyema, the nonsurgical treatment may be considered in patients in good neurosurgical condition with a GCS score of 15 and limited collection of pus on CT scans. Immediate neurosurgical treatment is still indicated in those with impaired consciousness, major focal deficits, or marked mass effect on CT scans.

\section{REFERENCES}

[1] Tsai, Y.D., Chang, W.N., Shen, C.C., Lin, Y.C., Lu, C.H., Liliang, P.C., et al. (2003) Intracranial suppuration: A clinical comparison of subdural empyemas and epidural abscesses. Surgical Neurology, 59, 191-196.
doi:10.1016/S0090-3019(02)01054-6

[2] Nathoo, N., Nadvi, S.S. and van Dellen, J.R. (1997) Infratentorial empyema: Analysis of 22 cases. Neurosurgery, 41, 1263-1269. doi:10.1097/00006123-199712000-00005

[3] Banerjee, A.D., Pandey, P., Devi, B.I., Sampath, S. and Chandramouli, B.A. (2009) Pediatric supratentorial subdural empyemas: A retrospective analysis of 65 cases. $P e-$ diatric Neurosurgery, 45, 11-18. doi:10.1159/000202619

[4] Keet, P.C. (1990) Cranial intradural abscess management of 641 patients during the 35 years from 1952 to 1986 . British Journal of Neurosurgery, 4, 273-278. doi:10.3109/02688699008992736

[5] Nathoo, N., Nadvi, S.S., van Dellen, J.R. and Gouws, E. (1999) Intracranial subdural empyemas in the era of computed tomography: A review of 699 cases. Neurosurgery, 44, 529-536. doi:10.1097/00006123-199903000-00055

[6] Venkatesh, M.S., Pandey, P., Devi, B.I., Khanapure, K., Satish, S., Sampath, S., et al. (2006) Pediatric infratentorial subdural empyema: Analysis of 14 cases. Journal of Neurosurgery, 105, 370-377.

[7] Bhandari, Y.S. and Sarkari, N.B.S. (1970) Subdural empyema. A review of 37 cases. Journal of Neurosurgery, 32, 35-39. doi:10.3171/jns.1970.32.1.0035

[8] Morgan, D.W. and Williams, B. (1985) Posterior fossa subdural empyema. Brain, 108, 983-992. doi:10.1093/brain/108.4.983

[9] Borovich, B., Johnson, E. and Spagnuolo, E. (1990) Infratentorial subdural empyema: Clinical and computerized tomography findings. Report of three cases. Journal of Neurosurgery, 72, 299-301. doi:10.3171/jns.1990.72.2.0299

[10] Kojima, A., Yamaguchi, N. and Okui, S. (2004) Supraand infratentorial subdural empyema secondary to septicemia in a patient with liverabscess-Case report. Neurologia Medico-Chirurgica (Tokyo), 44, 90-93. doi:10.2176/nmc.44.90

[11] Polyzoidis, K.S., Vranos, G., Exarchakos, G., Argyropoulou, M.I., Korantzopoulos, P. and Skevas, A. (2004) Subdural empyema and cerebellar abscess due to chronic otitis media. International Journal of Clinical Practice, 58, 214-217. doi:10.1111/j.1368-5031.2004.0050.x

[12] Sahjpaul, R.L. and Lee, D.H. (1999) Infratentorial subdural empyema, pituitary abscess, and septic cavernous sinus thrombophlebitis secondary to paranasal sinusitis: Case report. Neurosurgery, 44, 864-866. doi:10.1097/00006123-199904000-00101

[13] Singh, B. and Maharaj, T.J. (1993) Radical mastoidectomy: Its place in otitic intracranial complications. Journal of Laryngology \& Otology, 12, 1113-1118.

[14] Osman Farah, J., Kandasamy, J., May, P., Buxton, N. and Mallucci, C. (2009) Subdural empyema secondary to sinus infection in children. Child's Nervous System, 25, 199-205. doi:10.1007/s00381-008-0665-X

[15] Germiller, J.A., Monin, D.L., Sparano, A.M. and Tom, L.W. (2006) Intracranial complications of sinusitis in children and adolescents and their outcomes. Archives of Otolaryngology—Head and Neck Surgery, 132, 969-976. 
doi:10.1001/archotol.132.9.969

[16] Bannister, G., Williams, B. and Smith, S. (1981) Treatment of subdural empyema. Journal of Neurosurgery, 55, 82-88. doi:10.3171/jns.1981.55.1.0082

[17] Farmer, T.W. and Wise, G.R. (1973) Subdural empyema in infants, children and adults. Neurology, 23, 254-261. doi:10.1212/WNL.23.3.254

[18] Hitchcock, E. and Andreadis, A. (1964) Subdural empyema: A review of 29 cases. Journal of Neurology, Neurosurgery \& Psychiatry, 27, 422-434. doi:10.1136/jnnp.27.5.422

[19] Williams, B. (1983) Subdural empyema. Advances and Technical Standards in Neurosurgery, 9, 133-170. doi:10.1007/978-3-7091-7034-2_6

[20] Bok, A.P. and Peter, J.C. (1993) Subdural empyema: Burr holes or craniotomy? A retrospective computerized tomography-era analysis of treatment in 90 cases. Journal of Neurosurgery, 78, 574-578. doi:10.3171/jns.1993.78.4.0574

[21] Mauser, H.W., Ravijst, R.A., Elderson, A., van Gijn, J. and Tulleken, C.A. (1985) Nonsurgical treatment of subdural empyema. Case report. Journal of Neurosurgery, 63, 128-130. doi:10.3171/jns.1985.63.1.0128

[22] Rosazza, A., de Tribolet, N. and Deonna, T. (1979) Nonsurgical treatment of interhemispheric subdural empyemas. Helvetica Paediatrica Acta, 34, 577-581. 